\title{
Staggered Anisotropy Parameter Modification of the Anisotropic $t-J$ Model
}

\author{
T. Sedrakyan円 \\ Laboratoire d'Annecy-le-Vieux de Physique Théorique LAPTH \\ CNRS, UMR 5108, associée à l'Université de Savoie \\ BP 110, F-74941 Annecy-le-Vieux Cedex, France
}

\begin{abstract}
The anisotropic t-J model $\left(U_{q}(g l(2 \mid 1))\right.$ Perk-Schultz model) with staggered disposition of the anisotropy parameter along a chain is considered and the corresponding ladder type integrable model is constructed. This is a generalisation to spin- 1 case of the staggered $X X Z$ spin-1/2 model considered earlier. The corresponding Hamiltonian is calculated and, since it contains next to nearest neighbour interaction terms, can be written in a zig-zag form. The Algebraic Bethe Ansatz technique is applied and the eigenstates, along with eigenvalues of the transfer matrix of the model are found.
\end{abstract}

LAPTH-840/01

hep-th/0103027

February 2001

\footnotetext{
1e-mail:tigrans@moon.yerphi.am, Permanent address: Yerevan Physics Institute, Armenia
} 


\section{Introduction}

The interest to ladder type models was raised in a beginning of 90-s (see [1] for a review) in connection with high temperature superconductivity problems in metal oxides. It is believed that quasi-one dimensional multi-ladder chains of strongly interacting electrons reflect the most important aspects of two dimensional systems and also can reveal some properties of the weak coupling between conducting planes.

Recently there has been considerable interest in the construction of integrable ladder type models motivated by the desire to use powerful technique of Algebraic Bethe Ansatz (ABA) [2, 3] in the exact investigations of the variety of physical phases of the models.

In the articles [4] integrable ladder models were constructed by extension of the symmetry algebra, in [5] by defining first the ground state and then formulating a model, which has it. The higher conservation laws of an integrable models, which contains next to nearest neighbour interactions, were used in construction of ladder models in [6] by developing the approach of the article [7].The models with alternating spins were considered in [8, 9]. There are also some other attempts in this area [10, 11].

Usually integrable models are homogeneous along the chain, namely, the spectral $u$ and model parameters are the same in the product of $R$-matrices along the chain. It is obvious, that if one considers arbitrary shifts of the spectral parameters by some $z_{i}$ in the monodromy matrix we still have an integrable model. But in order to have a local Hamiltonian we need to consider shifts with fixed periodicity $n$, which causes the interaction of spins (or electrons) within an amount of $n$ neighbours, leading to $n$-ladder model. The staggered shift of the spectral parameter was first considered in [12 in an attempt to construct a relativistic invariant massive Tirring Model in a specific limit of the homogeneous $X X Z$-model.

In recent articles [13, 14] the authors have proposed a construction of some integrable chain models with $\mathbb{Z}_{2}$ grading of the states along the chain, as well as along the time directions. Hence, a way of constructing an integrable models with staggered inhomogeneity was proposed, motivated by the problems of three dimensional Ising model [16] and the Hall effect [30]. The inhomogeneous anisotropic $X X Z$ Heisenberg chain with staggered anisotropy parameter $\pm \Delta$ was constructed in [13], while the isotropic $t-J$ model was considered in [14.

The $X X Z$ and $t-J$ models with only inhomogeneous shift of the spectral parameter was previously considered in a chain of articles [18, 19, 20, 21], but these authors have not analysed the possibility of the inhomogeneity of the anisotropy parameter $\Delta$ (or other model parameters). As a result they have the same intertwining $R$-matrix as for ordinary homogeneous integrable $X X Z$ and $t-J$ models correspondingly and, therefore, the same quantum group structure behind. Contrary to this, in the construction presented in the articles [13] and [15] the integrable inhomogeneity appeared not only in shifts of the spectral parameter, but also in some structural changes. As a result we got modified Yang-Baxter equations (YBE), leading to a quantum group algebraic structure different 
from the usual $s l_{q}(2)$ [15].

As it is shown in the mentioned above articles, due to periodic shift of the spectral parameters the Hamiltonians of all this models contain at most next to nearest neighbour (NNN) interaction terms and therefore can be represented as integrable two leg ladder (zig-zag) models. The interaction between the legs of the ladder is represented in the Hamiltonian by a topological type terms written as the product of three neighbour spins and the anisotropic antisymmetric tensor as

$$
\hat{\epsilon}^{a b c} \sigma_{i}^{a} \sigma_{i+1}^{b} \sigma_{i+2}^{c} .
$$

As it was shown in [30], the model of free fermions hopping with inhomogeneous parameters, in a case when the rotational invariance is preserved, determines a Peierls type mechanism of mass generation, which is based on the breaking of the translational invariance for the translations of one lattice spacing. Perhaps the above mentioned 3-spin interaction terms are responsible for a gaped phase of the system, as it is discussed in the articles [22] in connection with high temperature superconductivity problems.

In this article, by use of the solution of the staggered YBE for the general $S l_{q}(n)$ quantum group presented in [15], we pass from spin- $1 / 2 X X Z$ model to three state $g l(2 \mid 1)$ supergroup based anisotropic $t-J$ model [23, 24, 25, 31] (or $U_{q}(g l(2 \mid 1))$ PerkSchultz model) with staggered sign of the anisotropy parameter. Following the technique of fermionization of spin models developed in [27] one can see, that anisotropic supersymmetric $t-J$ model cam be regarded as fermionized version of the anisotropic spin 1 bosonic Uimin-Lai-Sutherland model [28, 29, 17].

In Section 2 we present the solution of the staggered $Y B E$ for the $S l_{q}(1,2)$ case and calculate the Hamiltonian of the model in a ladder form.

In Section 3 we apply the technique of the Algebraic Bethe Ansatz $(A B A)$ [2, 3] and find the eigenvalues and eigenstates of our Hamiltonian. The Bethe equations $(B E)$ for the allowed values of the spectral parameters (momentums) are written down here.

\section{The Yang-Baxter Equations, their solution and the Hamiltonian of the Model}

In this Section we follow the technique developed in the articles [13, 14]. We modify the anisotropic supersymmetric $t-J$ model (which is the $U_{q}(g l(2 \mid 1))$ Perk-Schultz model [23, 24, 25]) in order to construct an integrable model with staggered disposition of the sign of the anisotropy parameter $\Delta$.

The principal ingredient of integrable models via Bethe Ansatz technique is the $R$ matrix. The $R_{a j}$-matrix acts as an intertwining operator on the direct product of the auxiliary $V_{a}(v)$ and quantum $V_{j}(u)$ spaces

$$
R_{a j}(u, v): V_{a}(u) \otimes V_{j}(v) \longrightarrow V_{j}(v) \otimes V_{a}(u) .
$$


For the anisotropic $t-J$ model the quantum and auxiliary spaces are three dimensional and corresponding $R$-matrix (see for example [24) can be defined by the following formula

$$
R_{a j}(z)=q z R_{a j, q}-q^{-1} z^{-1} R_{j a, q}^{-1},
$$

where we have introduced a multiplicative spectral parameter $z=e^{i u}$. The parameter $q$ defines the anisotropy of the model and, as it is shown in [25], the so called constant $R$-matrix $R_{q}$ is equal to

$$
R_{a j, q}=\lim _{z \rightarrow \infty} \frac{R(z)}{z}=\left(\begin{array}{lllllllll}
-1 & 0 & 0 & 0 & 0 & 0 & 0 & 0 & 0 \\
0 & -q^{-1} & 0 & 0 & 0 & 0 & 0 & 0 & 0 \\
0 & 0 & q^{-1} & 0 & 0 & 0 & 0 & 0 & 0 \\
0 & -q^{-1} \lambda & 0 & -q^{-1} & 0 & 0 & 0 & 0 & 0 \\
0 & 0 & 0 & 0 & -1 & 0 & 0 & 0 & 0 \\
0 & 0 & 0 & 0 & 0 & q^{-1} & 0 & 0 & 0 \\
0 & 0 & -q^{-1} \lambda & 0 & 0 & 0 & q^{-1} & 0 & 0 \\
0 & 0 & 0 & 0 & 0 & -q^{-1} \lambda & 0 & q^{-1} & 0 \\
0 & 0 & 0 & 0 & 0 & 0 & 0 & 0 & q^{-2}
\end{array}\right),
$$

with $\lambda=q-q^{-1}$.

It is very convenient for this model to consider its fermionized version by use of the technique developed in the article [26, 27]. Let us introduce two Fermi fields $c_{i, \sigma}, c_{i, \sigma}^{+}$with spin up and down states $\sigma=\uparrow, \downarrow$ at each site $i$ of the chain and three basic vectors of the corresponding spaces $V_{i}$ as follows

$$
|-\rangle \equiv|0, \downarrow\rangle, \quad|+\rangle \equiv|\uparrow, 0\rangle, \quad|0\rangle \equiv|0,0\rangle,
$$

numerated as $|1\rangle,|2\rangle,|3\rangle$ respectively. Therefore we have $\mathbb{Z}_{2}$ graded quantum $V_{j}(u)$ and auxiliary $V_{a}(v)$ spaces with the following parities

$$
p(|+\rangle)=p(|-\rangle)=1, \quad p(|0\rangle)=0 .
$$

Consider now Hubbard operators $X_{a_{2}}^{a_{1}}=\left|a_{2}\right\rangle\left\langle a_{1}\left|, X_{j_{2}}^{j_{1}}=\right| j_{2}\right\rangle\left\langle j_{1}\right|$, with $|a\rangle,|j\rangle$ defined as in formulas (5) and

$$
\begin{aligned}
X_{m}^{k}= & \left(\begin{array}{lll}
|-\rangle\langle-| & |-\rangle\langle+| & |-\rangle\langle 0| \\
|+\rangle\langle-| & |+\rangle\langle+| & |+\rangle\langle 0| \\
|0\rangle\langle-| & |0\rangle\langle+| & |0\rangle\langle 0|
\end{array}\right) \\
= & \left(\begin{array}{lll}
\left(1-n_{\uparrow}\right) n_{\downarrow} & c_{\downarrow}^{+} c_{\uparrow} & \left(1-n_{\uparrow}\right) c_{\downarrow}^{+} \\
c_{\uparrow}^{+} c_{\downarrow} & n_{\uparrow}\left(1-n_{\downarrow}\right) & c_{\uparrow}^{+}\left(1-n_{\downarrow}\right) \\
\left(1-n_{\uparrow}\right) c_{\downarrow} & c_{\uparrow}\left(1-n_{\downarrow}\right) & \left(1-n_{\uparrow}\right)\left(1-n_{\downarrow}\right)
\end{array}\right),
\end{aligned}
$$

where $n_{\sigma}=c_{\sigma}^{+} c_{\sigma}$ is the particle number operator. The demand that the trace of this operator acts on auxiliary and quantum spaces as the identity operator means that the double occupancy of the sites by fermions is excluded

$$
\Delta|l\rangle=X_{m}^{m}|l\rangle=\left(1-n_{\uparrow} n_{\downarrow}\right)|l\rangle=|l\rangle, \quad m, l=1,2,3 .
$$


Now we can write down the formula connecting the fermionic $R_{a j}$-operator of the anisotropic supersymmetric $T-J$ model with the $R_{a j}$-matrix defined by the expression-s (通) and (4)

$$
R_{a j}=R_{a j}\left|j_{1}\right\rangle\left|a_{1}\right\rangle\left\langle a_{1}\right|\left\langle j_{1}\right|=(-1)^{p\left(a_{1}\right) p\left(j_{2}\right)}\left(R_{a j}\right)_{a_{1} j_{1}}^{a_{2} j_{2}} X_{a_{2}}^{a_{1}} X_{j_{2}}^{j_{1}} .
$$

It is straightforward to check that this fermionic $R$-operator satisfies the YBE in the operator form [27.

Let us now consider $\mathbb{Z}_{2}$ graded quantum $V_{j, \rho}(v)$ and auxiliary $V_{a, \sigma}(u)$ spaces, $\rho, \sigma=0,1$. In this case we have $4 \times 4 R$-matrices, which act on the direct product of the spaces $V_{a, \sigma}(u)$ and $V_{j, \rho}(v),(\sigma, \rho=0,1)$, mapping them on the intertwined direct product of $V_{a, \bar{\sigma}}(u)$ and $V_{j, \bar{\rho}(v)}$ with the complementary $\bar{\sigma}=(1-\sigma), \bar{\rho}=(1-\rho)$ indices

$$
R_{a j, \sigma \rho}(u, v): \quad V_{a, \sigma}(u) \otimes V_{j, \rho}(v) \rightarrow V_{j, \bar{\rho}}(v) \otimes V_{a, \bar{\sigma}}(u) .
$$

It is convenient to introduce two transmutation operations $\iota_{1}$ and $\iota_{2}$ with the property $\iota_{1}^{2}=\iota_{2}^{2}=i d$ for the quantum and auxiliary spaces correspondingly, and to mark the operators $R_{a j, \sigma \rho}$ as follows

$$
\begin{array}{rlrl}
R_{a j, 00} & \equiv R_{a j}, & & R_{a j, 01} \equiv R_{a j}^{\iota_{1}}, \\
R_{a j, 10} \equiv R_{a j}^{\iota_{2}}, & & R_{a j, 11} \equiv R_{a j}^{\iota_{1} \iota_{2}} .
\end{array}
$$

The introduction of the $\mathbb{Z}_{2}$ grading in quantum space means, that we have now two monodromy matrices $M_{\rho}, \rho=0$, 1 , which act on the space $V_{\rho}=\prod_{j=1}^{N} V_{j, \rho}$ by mapping it on $V_{\bar{\rho}}=\prod_{j=1}^{N} V_{j, \bar{\rho}}$

$$
M_{\rho} \quad: V_{\rho} \rightarrow V_{\bar{\rho}}, \quad \rho=0,1 .
$$

It is clear now, that the monodromy matrix of the model, which should define the partition function, is the product of two monodromy matrices

$$
M(u)=M_{0}(u) M_{1}(u) .
$$

Now, because of the grading in the auxiliary space, we would like to construct the monodromy matrices $M_{0,1}$ as a staggered product of the $R_{a j}$ and $\bar{R}_{a j}^{\iota_{2}}$ matrices. Let us define

$$
\begin{aligned}
M_{0}(u) & =\prod_{j=1}^{N} R_{a, 2 j-1}(u) \bar{R}_{a, 2 j}^{\iota_{2}}(u) \\
M_{1}(u) & =\prod_{j=1}^{N} \bar{R}_{a, 2 j-1}^{\iota_{1}}(u) R_{a, 2 j}^{\iota_{1} \iota_{2}}(u),
\end{aligned}
$$

where the notation $\bar{R}$ in general means the different parametrisation of the $R$-matrix via model $(\lambda)$ and spectral $(u)$ parameters and can be considered as an operation over $R$ with property $\overline{\bar{R}}=R$. 
In order to have a integrable model with commuting transfer matrices

$$
\tau(u)=\operatorname{tr}_{0} \operatorname{tr}_{0^{\prime}} M(u)=\tau_{0}(u) \tau_{1}(u) .
$$

for different spectral parameters

$$
[\tau(u), \tau(v)]=0
$$

it is enough to have the following relations for the $\tau_{\sigma}(u)=\operatorname{tr} M_{\sigma}(u), \quad(\sigma=0,1)$

$$
\tau_{\sigma}(\lambda, u) \tau_{1-\sigma}(\lambda, v)=\bar{\tau}_{\sigma}(\lambda, v) \bar{\tau}_{1-\sigma}(\lambda, u), \quad \sigma=0,1
$$

It is not hard to see, that in order to ensure the commutativity condition (16) the $R$ - and $\bar{R}$-matrices in the expression (14) should fulfill the following two Yang-Baxter Equations, which in so called check formalism defined by operator $\check{R}_{i j}=R_{i j} P_{i j}$, $\left(P_{i j}\right.$ is the permutation operator), has the form

$$
\check{R}_{12}(u, v) \check{\bar{R}}_{23}^{\iota_{1}}(u) \check{R}_{12}(v)=\check{R}_{23}^{\iota_{1}}(v) \check{\bar{R}}_{12}(u) \check{\tilde{R}}_{23}(u, v)
$$

and

$$
\check{\tilde{R}}_{12}(u, v) \check{R}_{23}^{\iota_{1} \iota_{2}}(u) \check{\bar{R}}_{12}^{\iota_{2}}(v)=\check{\bar{R}}_{23}^{\iota_{1} \iota_{2}}(v) \check{R}_{12}^{\iota_{2}}(u) \check{R}_{23}(u, v) .
$$

If the $\iota_{1}$ and $\iota_{2}$ operations are trivial this $Y B E$ 's are reducing to ordinary ones. Therefore one can take an integrable model with the $R$-matrix fulfilling $Y B E$ and try to find nontrivial $\iota_{1}$ and $\iota_{2}$ operations. For the $S l_{q}(N)$ case this type of solutions were found in [15] from where we will extract the solution for the $S l_{q}(2 \mid 1)$ case under consideration. The solution of the first set of equations (18) with the nontrivial $\iota_{1}$ operation looks like

$$
\check{R}^{\iota_{1}}(z)=q z \check{R}_{q}^{\iota_{1}}-q^{-1} z^{-1}\left(\check{R}_{q}^{\iota_{1}}\right)^{-1}
$$

with

$$
\check{R}_{q}^{\iota_{1}}=\tilde{R}_{q}=\left(\begin{array}{lllllllll}
-1 & 0 & 0 & 0 & 0 & 0 & 0 & 0 & 0 \\
0 & -q^{-1} \lambda & 0 & -q^{-1} & 0 & 0 & 0 & 0 & 0 \\
0 & 0 & -q^{-1} \lambda & 0 & 0 & 0 & -q^{-1} & 0 & 0 \\
0 & -q^{-1} & 0 & 0 & 0 & 0 & 0 & 0 & 0 \\
0 & 0 & 0 & 0 & -1 & 0 & 0 & 0 & 0 \\
0 & 0 & 0 & 0 & 0 & -q^{-1} \lambda & 0 & -q^{-1} & 0 \\
0 & 0 & -q^{-1} & 0 & 0 & 0 & 0 & 0 & 0 \\
0 & 0 & 0 & 0 & 0 & -q^{-1} & 0 & 0 & 0 \\
0 & 0 & 0 & 0 & 0 & 0 & 0 & 0 & q^{-2}
\end{array}\right) .
$$

and the bar operation in (18, 19) is a multiplicative shift of the multiplicative spectral parameter $z=e^{i u}$ by the new model parameter $h=e^{i \theta}$ as $\check{R}(\bar{z})=\check{R}\left(e^{i \bar{u}}\right)=\check{R}\left(h z^{-1}\right)$. 
This definition for the $\iota_{1}$ operation means that

$$
\left(R_{q}^{\iota_{1}}\right)_{13}^{13}=-\left(R_{q}\right)_{13}^{13}, \quad\left(R_{q}^{\iota_{1}}\right)_{23}^{23}=-\left(R_{q}\right)_{23}^{23}, \quad\left(R_{q}^{\iota_{1}}\right)_{12}^{12}=-\left(R_{q}\right)_{12}^{12} .
$$

If we define the operation $\iota_{2}$ on $\check{R}(z)$ as

$$
\check{R}^{\iota_{2}}(z)=\check{R}^{\iota_{1}}\left(z^{-1}\right),
$$

then easy to see that second set of YBEs will coincide with the first set of $Y B E$-s.

The explicit expressions for the matrix elements of $R(z)$ and $R^{\iota_{1}}(z)$ matrices which are fulfilling the $Y B E$-s (18-19) are the followings

$$
\begin{aligned}
& R_{11}^{11}(z)=R_{22}^{22}(z)=q^{-1} z^{-1}-q z, \quad R_{33}^{33}(z)=q^{-1} z-q z^{-1}, \\
& R_{11}^{33}(z)=R_{22}^{33}(z)=R_{33}^{11}(z)=R_{33}^{22}(z)=-R_{11}^{22}(z)=-R_{22}^{11}(z)=z-z^{-1} \\
& R_{21}^{12}(z)=R_{31}^{13}(z)=R_{32}^{23}(z)=-z \lambda, \quad R_{12}^{21}(z)=R_{13}^{31}(z)=R_{23}^{32}(z)=-z^{-1} \lambda
\end{aligned}
$$

and

$$
\begin{aligned}
& \left(R^{\iota_{1}}(z)\right)_{11}^{11}=\left(R^{\iota_{1}}(z)\right)_{22}^{22}=q^{-1} z^{-1}-q z, \quad\left(R^{\iota_{1}}(z)\right)_{33}^{33}=q^{-1} z-q z^{-1} \\
& \left(R^{\iota_{1}}(z)\right)_{11}^{33}=\left(R^{\iota_{1}}(z)\right)_{22}^{33}=\left(R^{\iota_{1}}(z)\right)_{33}^{11}=\left(R^{\iota_{1}}(z)\right)_{33}^{22}=\left(R^{\iota_{1}}(z)\right)_{11}^{22}=\left(R^{\iota_{1}}(z)\right)_{22}^{11}=-z+z^{-1} \\
& \left(R^{\iota_{1}}(z)\right)_{21}^{12}=\left(R^{\iota_{1}}(z)\right)_{31}^{13}=\left(R^{\iota_{1}}(z)\right)_{32}^{23}=-z \lambda, \\
& \left(R^{\iota_{1}}(z)\right)_{12}^{21}=\left(R^{\iota_{1}}(z)\right)_{13}^{31}=\left(R^{\iota_{1}}(z)\right)_{23}^{32}=-z^{-1} \lambda
\end{aligned}
$$

Therefore we have found an integrable inhomogeneous model and our aim now is to write down the expression for the Hamiltonian. But in order to have a local Hamiltonian it is necessary to have a point $u_{0}$, where

$$
\check{R}_{i j}\left(u_{0}\right)=\text { const. } \hat{I}_{i j},
$$

with $\hat{I}_{i j}$ being an identity operator.

¿From the form of $\check{R}$-matrix (20) one can easily see that the value $u_{0}=0$ (or $z_{0}=$ $e^{i u_{0}}=1$ ) is just the needed one. Let us notice that though $\left.\check{R}_{i j}(u)\right|_{u=0}=-\lambda \hat{I}_{i j}$, we have $\left.\check{R}_{i j}(\bar{u})\right|_{u=0} \neq \hat{I}_{i j}$. Because of this, and as future calculations will show, the Hamiltonian of our model contains, together with nearest-neighbour interaction terms, also next-to nearest-neighbour interaction terms.

By definition the Hamiltonian of the model is the logarithmic derivative of the transfer matrix at that point

$$
\mathfrak{H}=-\left.\frac{\partial \ln \tau(u)}{\partial u}\right|_{u=0}
$$


For calculations of logarithmic derivative of the Transfer matrix (15) we need to insert the linear expansions of the operators $\check{R}(z), \check{R}^{\iota_{1} \iota_{2}}(z), \check{R}^{\iota_{1}}(\bar{z}), \check{R}^{\iota_{2}}(\bar{z})$ around the point $u=0$

$$
\begin{array}{rlr}
\check{R}(u) & =-\lambda+u H, \quad H=i q \check{R}_{q}+i q^{-1} \check{R}_{q}^{-1}, \\
\check{R}(\bar{u}) & =\check{R}(h)+u \bar{H}, \quad \bar{H}=-i q h \check{R}_{q}-i q^{-1} h^{-1} \check{R}_{q}^{-1}, \\
\check{R}^{\iota_{1}}(\bar{u}) & =\check{R}^{\iota_{1}}(h)+u \bar{H}^{\iota_{1}}, \quad \bar{H}^{\iota_{1}}=-i q h \check{R}_{q}^{\iota_{1}}-i q^{-1} h^{-1}\left(\check{R}_{q}^{\iota_{1}}\right)^{-1}, \\
\check{R}^{\iota_{2}}(\bar{u}) & =\check{R}^{\iota_{1}}\left(h^{-1}\right)+u \bar{H}^{\iota_{2}}, \quad \bar{H}^{\iota_{2}}=i q h^{-1} \check{R}_{q}^{\iota_{1}}+i q^{-1} h\left(\check{R}_{q}^{\iota_{1}}\right)^{-1}, \\
\check{R}^{\iota_{1} \iota_{2}}(u) & =-\lambda-u H
\end{array}
$$

into the expressions of the Monodromy matrix

$$
M(u)=\check{R}_{01}^{\iota_{2}}(\bar{u}) \check{R}_{12}(u) \check{R}_{23}^{\iota_{2}}(\bar{u}) \cdots \check{R}_{01}^{\iota_{1}}(\bar{u}) \check{R}_{12}^{\iota_{1} \iota_{2}}(u) \check{R}_{23}^{\iota_{1}}(\bar{u}) \cdots
$$

and extract the linear terms in $u$ from the product. By taking into account that

$$
\check{R}^{\iota_{1}}(h) \check{R}^{\iota_{1}}\left(h^{-1}\right)=\left(\lambda^{2}+4 \sin ^{2} \theta\right) \hat{I},
$$

with $\theta=-i \log h$ as an additive shift of the spectral parameter, we finally obtain

$$
\begin{aligned}
& -i \lambda\left(\lambda^{2}+4 \sin ^{2} \theta\right) H_{j-1, j, j+1}= \\
& =(-1)^{j-1} \sum_{\sigma=\uparrow, \downarrow}\left\{c_{j-1, \sigma}^{+} c_{j+1, \sigma}\left[\left(1-n_{j, \bar{\sigma}}\right) f_{4}\left(q, h^{(-1)^{j}}\right)+n_{j, \bar{\sigma}} f_{0}\left(q, h^{(-1)^{j}}\right)\right]\right. \\
& \left.+c_{j+1, \sigma}^{+} c_{j-1, \sigma}\left[\left(1-n_{j, \bar{\sigma}}\right) f_{4}\left(q, h^{-(-1)^{j}}\right)+n_{j, \bar{\sigma}} f_{0}\left(q, h^{-(-1)^{j}}\right)\right]\right\} \\
& -(-1)^{j-1}\left\{S_{j-1}^{\mu} S_{j+1}^{\mu}\left[\left(1-n_{j}\right) f_{0}(q, h)-n_{j} \frac{f_{4}(q, h)+f_{4}\left(q, h^{-1}\right)}{2}\right]\right. \\
& \left.-\quad\left[S_{j-1}^{3} S_{j+1}^{3}+\frac{1}{4} n_{j-1} n_{j+1}\right]\left(2 n_{j}-1\right) f_{6}(q, h)\right\}+(-1)^{j-1} \sum_{\sigma=\uparrow, \downarrow}\left\{c_{j, \sigma}^{+} c_{j+1, \sigma}\right. \\
& +\quad\left[\left(1-n_{j+2, \bar{\sigma}}\right) f_{j(\bmod 2)+1}(q, h)+(-1)^{j}\left(1-n_{j-1, \bar{\sigma}}\right) f_{j(\bmod 2)+1}\left(q, h^{-1}\right)\right] \\
& +c_{j+1, \sigma}^{+} c_{j, \sigma}\left[\left(1-n_{j-1, \bar{\sigma}}\right) f_{j(\bmod 2)+1}(q, h)+(-1)^{j}\left(1-n_{j+2, \bar{\sigma}} f_{j(\bmod 2)+1}\left(q, h^{-1}\right)\right]\right\} \\
& +\left[S_{2 j-1}^{\mu} S_{2 j}^{\mu} \frac{f_{2}(q, h)+f_{2}\left(q, h^{-1}\right)}{2}+f_{6}(q, h) n_{2 j-1} n_{2 j}\right]\left(n_{2 j-2}-n_{2 j+1}\right) \\
& +2 S_{j-1}^{a} S_{j}^{b} S_{j+1}^{c} \hat{\epsilon}^{a b c}+(-1)^{j+1} \sum_{\{k l m\}=\{j-1, j, j+1\}} S_{k}^{\mu} S_{l m}^{\mu} \hat{\epsilon}^{k l m} f_{5}(q, h) \\
& +f_{3}(q, h)\left(n_{j-1} n_{j}+n_{j} n_{j+1}\right)
\end{aligned}
$$

where $\mu=1,2$.

In this expression for the Hamiltonian the spin variables are as in usual $t-J$ model

$$
S^{+}=c_{\uparrow}^{+} c_{\downarrow}, \quad S^{-}=c_{\downarrow}^{+} c_{\uparrow}, \quad S^{3}=\frac{n_{\uparrow}-n_{\downarrow}}{2},
$$


the anisotropic antisymmetric tensors $\hat{\epsilon}^{a b c}, \quad a, b, c=1,2,3$

and $\hat{\epsilon}^{k l m}, \quad k, l, m,=j-1, j, j+1$ are defined as follows

$$
\begin{aligned}
& \hat{\epsilon}^{a 3 b}=f_{1}\left(q^{1 / 2}, h^{2}\right) \epsilon^{a 3 b} \\
& \hat{\epsilon}^{3 a b}=\hat{\epsilon}^{a b 3}=f_{1}(q, h) \epsilon^{3 a b}
\end{aligned} \quad \hat{\epsilon}^{k l m}= \begin{cases}-\hat{\epsilon}^{l k m} & \text { if }|k-l|=2, \\
\hat{\epsilon}^{l k m} & \text { if }|k-l|=1\end{cases}
$$

where $\epsilon^{a b c}$ is ordinary antisymmetric tensor, and for functions $f_{r}(q, h)$ we got

$$
\begin{aligned}
& f_{0}(q, z)=2\left(h-h^{-1}\right)^{2}, \\
& f_{1}(q, z)=2\left(h-h^{-1}\right)\left(q^{2}-q^{-2}\right), \\
& f_{3}(q, z)=4\left(h^{-1}-h\right)\left(3 q h+q h^{-1}-q^{-1} h-3 q^{-1} h^{-1}\right), \\
& f_{4}(q, z)=2\left(q^{1 / 2} h^{-1}-q^{-1 / 2} h\right)^{2}-2\left(q^{1 / 2}-q^{-1 / 2}\right)^{2}, \\
& f_{5}(q, z)=2\left(q-q^{-1}\right)\left(h-h^{-1}\right), \\
& f_{6}(q, z)=2\left(q+q^{-1}\right)\left(h-h^{-1}\right)^{2} .
\end{aligned}
$$

It is convenient now to write the Hamiltonian (31) in a ladder form represented graphically as in Figure 11.

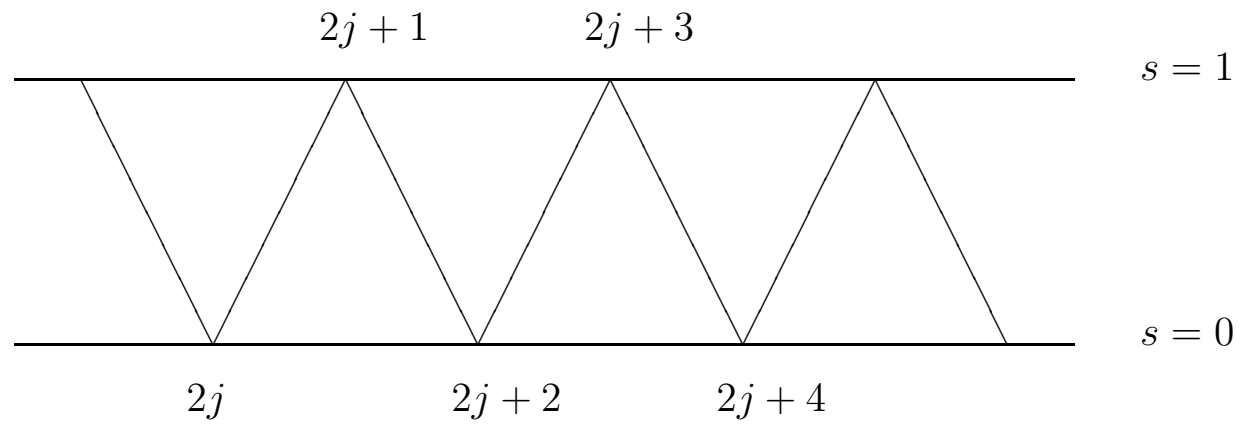

Figure 1: Zig-zag ladder chain

Let us consider the even $(2 j)$ and the odd $(2 j+1)$ points of the chain as a sites $(j)$ of two different chains labeled by $s=0$ and 1 correspondingly. The Fermi fields will be marked now as

$$
c_{j, s}=c_{2 j+s}, \quad s=0,1
$$

With these notations it is straightforward to obtain from the expression (31) the following ladder Hamiltonian

$$
\begin{aligned}
& -i \lambda\left(\lambda^{2}+4 \sin ^{2} \theta\right) H_{j, s}=H_{j, s}^{c}+H_{j, s}^{r}+H_{j, s}^{3 S}+H_{j, s}^{h S} \\
& =(-1)^{s} \sum_{\sigma=\uparrow, \downarrow}\left\{c_{j, s, \sigma}^{+} c_{j+1, s, \sigma}\left[\left(1-n_{j+s, s+1, \bar{\sigma}}\right) f_{4}\left(q, h^{(-1)^{s+1}}\right)+n_{j+s, s+1, \bar{\sigma}} f_{0}\left(q, h^{(-1)^{s+1}}\right)\right]\right. \\
& \left.+\quad c_{j+1, s, \sigma}^{+} c_{j, s, \sigma}\left[\left(1-n_{j+s, s+1, \bar{\sigma}}\right) f_{4}\left(q, h^{-(-1)^{s}}\right)+n_{j+s, s+1, \bar{\sigma}} f_{0}\left(q, h^{-(-1)^{s}}\right)\right]\right\}
\end{aligned}
$$




$$
\begin{aligned}
& -(-1)^{s}\left\{S_{j, s}^{\mu} S_{j+1, s}^{\mu}\left[\left(1-n_{j+s, s+1}\right) f_{0}(q, h)-n_{j+s, s+1} \frac{f_{4}(q, h)+f_{4}\left(q, h^{-1}\right)}{2}\right]\right. \\
& \left.-\quad\left[S_{j, s}^{3} S_{j+1, s}^{3}+\frac{1}{4} n_{j, s} n_{j+1, s}\right]\left(2 n_{j+s, s}-1\right) f_{6}(q, h)\right\}+(-1)^{j-1} \sum_{\sigma=\uparrow, \downarrow}\left\{c_{j, s, \sigma}^{+} c_{j+s, s+1, \sigma}\right. \\
& \cdot \quad\left[\left(1-n_{j+1, s, \bar{\sigma}}\right) f_{s+1}\left(q, h^{(-1)^{s}}\right)+(-1)^{s}\left(1-n_{j-1+s, s+1, \bar{\sigma}}\right) f_{s+1}\left(q, h^{(-1)^{s+1}}\right)\right] \\
& +\quad c_{j+s, s+1, \sigma}^{+} c_{j, s, \sigma}\left[\left(1-n_{j+1, s, \bar{\sigma}}\right) f_{s+1}\left(q, h^{(-1)^{s+1}}\right)+(-1)^{s}\left(1-n_{j-1+s, s+1, \bar{\sigma}} f_{s+1}\left(q, h^{(-1)^{s}}\right)\right]\right\} \\
& +\quad\left[S_{j, 1}^{\mu} S_{j+1,0}^{\mu} \frac{f_{2}(q, h)+f_{2}\left(q, h^{-1}\right)}{2}+f_{6}(q, h) n_{j, 1} n_{j+1,0}\right]\left(n_{j, 0}-n_{j+1,1}\right) \\
& +\quad 2 S_{j, s}^{a} S_{j+s, s+1}^{b} S_{j+1, s}^{c} \hat{\epsilon}^{a b c}+(-1)^{s} \quad S_{k}^{\mu} S_{l m}^{\mu} \hat{\epsilon}^{k l m} f_{5}(q, h) \\
& +\quad f_{3}(q, h) n_{j, s} n_{j+s, s+1} \quad(36)=\{(j, s),(j+s, s+1),(j+1, s)\}
\end{aligned}
$$

Though this expression is big but one can recognise four clear terms there. The first one is the Hamiltonian of anisotropic $t-J$ model for the each of chains (marked as $H_{j s}^{c}$ ). The second is anisotropic $t-J$ term for the rungs $\left(H_{j a}^{r}\right)$. The third term $\left(H_{j s}^{3 S}\right)$, which was discussed in the introduction, is written for the each triangle of the zig-zag, represents the interaction between chains and has a topological form. The last term contains spin-spin Heisenberg interaction together with hopping of fermions and can be thought as spin-orbit interaction.

At the end of this Section let us write the $q \rightarrow 1$ limit of this Hamiltonian, which corresponds to isotropic case. In this limit it is necessary to rescale the additive spectral parameter $u$ and introduce $w=\log z / \log q$ together with new additional model parameter $\theta=\log z / \log q$, which should be kept finite. Then we will obtain

$$
\begin{aligned}
\frac{(-1)^{s+1}}{2} H_{j s} & =\sum_{\sigma=\uparrow, \downarrow} c_{j, s, \sigma}^{+} c_{j+1 . s, \sigma}+c_{j+1, s, \sigma}^{+} c_{j . s, \sigma} \\
& +\left[S_{j, s}^{\mu} S_{j+1, s}^{\mu}+S_{j, s}^{3} S_{j+1, s}^{3}+\frac{1}{4} n_{j, s} n_{j+1, s}\right]\left(2 n_{j+s, s+1}-1\right) \\
& -n_{j, s} n_{j+s, s+1} n_{j+1 . s}-(-1)^{s} 5 n_{j, s} n_{j+s, s+1},
\end{aligned}
$$

which looks like an ordinary supersymmetric $t-J$ model for each of chains. But the presence of the factor $2 n-1$ in front of spin-spin interaction means that it is ferromagnetic or antiferromagnetic regarding whether the corresponding site in the other chain is occupied by fermion or not.

\section{$3 A B A$ solution of the model}

The $A B A$ solution for the homogeneous Perk-Schultz model was carried out in [24] and in a chain of articles [31] for a model with open boundaries. In this section we will 
use the technique of Algebraic Bethe Ansatz ([2]-[3]) in order to find the eigenstates and eigenvalues of the Hamiltonian (31) for staggered inhomogeneity.

At the beginning we need the definition of the $L$-operator

$$
L_{i, j}(z)_{m}^{m^{\prime}}=\left\langle m\left|R_{i, j}(z)\right| m^{\prime}\right\rangle=(-1)^{p\left(m^{\prime}\right) p\left(n^{\prime}\right)}\left(R_{i j}(z)\right)_{m n}^{m^{\prime} n^{\prime}} X_{n^{\prime}}^{n}
$$

which is a $3 \times 3$ matrix in a horizontal auxiliary space, with the matrix elements acting in quantum space. It has the following expression

$$
\begin{gathered}
\left(L_{i j}\right)_{m}^{m^{\prime}}(z)= \\
\left(\begin{array}{ccc}
\left(q z-q^{-1} z^{-1}\right)\left(1-n_{\uparrow}\right) n_{\downarrow} & \\
+\left(z-z^{-1}\right)\left(1-n_{\downarrow}\right) & \lambda z^{-1} c_{\downarrow}^{+} c_{\uparrow} & \\
& \left(z-z^{-1}\right)\left(1-n_{\uparrow}\right) n_{\downarrow} & \\
\lambda z c_{\uparrow}^{+} c_{\downarrow} & -\left(-q z+z^{-1} z^{-1}\right) n_{\uparrow}\left(1-n_{\downarrow}\right) & -\lambda z^{-1} c_{\uparrow}^{+}\left(1-n_{\downarrow}\right) \\
& +\left(z-z^{-1}\right)\left(1-n_{\uparrow}\right)\left(1-n_{\downarrow}\right) & \\
& & \left(z-z^{-1}\right)\left(1-n_{\uparrow}\right) n_{\downarrow} \\
& & +\left(z-z^{-1}\right) n_{\uparrow}\left(1-n_{\downarrow}\right)+ \\
& & \left(q^{-1} z-q z^{-1}\right)\left(1-n_{\uparrow}\right)\left(1-n_{\downarrow}\right)
\end{array}\right)
\end{gathered} .
$$

The matrix elements of the monodromy operator (13) between auxiliary states $\left|k^{\prime}\right\rangle$ and $\langle k|$ can be expressed as a product of $L_{i j}$ matrices

$$
\begin{aligned}
& M_{0}(z)_{k^{\prime}}^{k}=\left\langle k\left|M_{0}(z)\right| k^{\prime}\right\rangle=L_{01}^{\iota_{2}}(\bar{z})_{k_{1}}^{k} L_{02}(z)_{k_{2}}^{k_{1}} \cdots L_{0 N}(z)_{k^{\prime}}^{k_{N-1}}, \\
& M_{1}(z)_{k^{\prime}}^{k}=\left\langle k\left|M_{1}(z)\right| k^{\prime}\right\rangle=L_{01}^{\iota_{1} \iota_{2}}(z)_{k_{1}}^{k} L_{02}^{\iota_{1}}(\bar{z})_{k_{2}}^{k_{1}} \cdots L_{0 N}^{\iota_{1}}(\bar{z})_{k^{\prime}}^{k_{N-1}}
\end{aligned}
$$

which in matrix form looks like

$$
M_{s}(z)_{k^{\prime}}^{k}=\left(\begin{array}{lll}
A_{s, 11}(z) & A_{s, 12}(z) & B_{s, 1}(z) \\
A_{s, 12}(z) & A_{s, 22}(z) & B_{s, 2}(z) \\
C_{s, 1}(z) & C_{s, 2}(z) & D_{s}(z)
\end{array}\right), \quad s=0,1
$$

where $A_{s, a b}, B_{s, a}, C_{s, a}, D_{s} ;(a, b=1,2)$ are operators in the the quantum space.

The graded property of the model is the origin of the following form of the transfer matrix (15)

$$
\tau_{s}(z)=-A_{s, 11}(z)-A_{s, 22}(z)+D_{s}(z), \quad s=0,1 .
$$

Now we would like to take the empty fermionic state

$$
|\Omega\rangle_{s}=|0,0, \ldots, 0\rangle_{s}=|0\rangle_{1 s}|0\rangle_{2 s} \cdots|0\rangle_{N s}, \quad s=0,1
$$


as a "test" vacuum and let us demonstrate that the "vacuum" $|\Omega\rangle$ is indeed eigenstate of the transfer matrix (42)

$$
\tau_{s}(z)|\Omega\rangle_{s}=\nu_{s}^{(0)}|\Omega\rangle_{1-s}
$$

The action of $L_{0 k}(z)$ and $L_{0 k}^{\iota_{2}}(\bar{z})$ on the $k$-th empty state $|0\rangle_{k}$ is easy to calculate

$$
\begin{aligned}
L_{0 k}(z)_{m}^{m^{\prime}}|0\rangle & =\left(\begin{array}{lll}
z-z^{-1} & 0 & -\lambda z^{-1} c_{\downarrow}^{+} \\
0 & z-z^{-1} & -\lambda z^{-1} c_{\uparrow}^{+} \\
0 & 0 & q^{-1} z-q z^{-1}
\end{array}\right)|0\rangle_{k} \\
L_{0 k}^{\iota_{2}}(\bar{z})_{m}^{m^{\prime}}|0\rangle & =\left(\begin{array}{lll}
-h^{-1} z+h z^{-1} & 0 & \lambda h z^{-1} c_{\downarrow}^{+} \\
0 & -h^{-1} z+h z^{-1} & \lambda h z^{-1} c_{\uparrow}^{+} \\
0 & 0 & h^{-1} q^{-1} z-h q z^{-1}
\end{array}\right)|0\rangle_{k},
\end{aligned}
$$

and as we see it has an upper-triangular form. Because the upper triangular matrices form a semigroup the action of the monodromy matrix $M_{0}(z)_{k^{\prime}}^{k}$ on $|\Omega\rangle_{0}$ also have an upper-triangular form directly following from the expression (40)

$$
\begin{aligned}
& M_{0}(z)_{m}^{m^{\prime}}|\Omega\rangle_{0}= \\
& \left(\begin{array}{lll}
\left(-h^{-1} z+h z^{-1}\right)^{\frac{N}{2}} & 0 & B_{0,1} \\
\left(z-z^{-1}\right)^{\frac{N}{2}} & & \\
0 & \left(-h^{-1} z+h z^{-1}\right)^{\frac{N}{2}}\left(z-z^{-1}\right)^{\frac{N}{2}} & B_{0,2} \\
0 & 0 & \left(h^{-1} q^{-1} z-h q z^{-1}\right)^{\frac{N}{2}} \\
0 & & \left(q^{-1} z-q z^{-1}\right)^{\frac{N}{2}}
\end{array}\right)|\Omega\rangle_{0} .
\end{aligned}
$$

Following the definitions (21-23) of the $\iota_{1}$ and $\iota_{2}$ operations one can easily find the actions of $L_{0 k}^{\iota_{1} \iota_{2}}(z)$ and $L_{0 k}^{\iota_{1}}(\bar{z})$ on $|0\rangle_{k}$ by use of formulas

$$
L^{\iota_{1} \iota_{2}}(z)=L\left(z^{-1}\right), \quad L^{\iota_{1}}(\bar{z})=L^{\iota_{2}}\left(\bar{z}^{-1}\right),
$$

and correspondingly the action of $M_{1}(z)_{k^{\prime}}^{k}$ on $|\Omega\rangle_{1}$ will be

$$
\begin{aligned}
& M_{1}(z)_{m}^{m^{\prime}}|\Omega\rangle_{1}= \\
& \left(\begin{array}{lll}
\left(h^{-1} z-h z^{-1}\right)^{\frac{N}{2}} & 0 & B_{1,1} \\
\left(-z+z^{-1}\right)^{\frac{N}{2}} & & \\
0 & \left(h^{-1} z-h z^{-1}\right)^{\frac{N}{2}}\left(-z+z^{-1}\right)^{\frac{N}{2}} & B_{1,2} \\
0 & 0 & \left(h q^{-1} z^{-1}-h^{-1} q z\right)^{\frac{N}{2}} \\
& & \left(q^{-1} z^{-1}-q z\right)^{\frac{N}{2}}
\end{array}\right)|\Omega\rangle_{1}
\end{aligned}
$$


Now we see that $|\Omega\rangle$ is the eigenstate of $\tau(u)$ with the eigenvalue $\nu^{(0)}(z)$

$$
\begin{aligned}
& \nu^{(0)}(z)=\nu_{0}^{(0)}(z) \nu_{1}^{(0)}(z), \\
& \nu_{0}^{(0)}(z)=-2\left(-h^{-1} z+h z^{-1}\right)^{\frac{N}{2}}\left(z-z^{-1}\right)^{\frac{N}{2}}+\left(h^{-1} q^{-1} z-h q z^{-1}\right)^{\frac{N}{2}}\left(q^{-1} z-q z^{-1}\right)^{\frac{N}{2}}, \\
& \nu_{1}^{(0)}(z)=-2\left(h^{-1} z-h z^{-1}\right)^{\frac{N}{2}}\left(-z+z^{-1}\right)^{\frac{N}{2}}+\left(h q^{-1} z^{-1}-h^{-1} q z\right)^{\frac{N}{2}}\left(q^{-1} z^{-1}-q z\right)^{\frac{N}{2}},
\end{aligned}
$$

which follows from the expressions (15), (42), (44), (47). Simultaneously we see, that the $B_{s, 1}(z)$ and $B_{s, 2}(z)(s=0,1)$ operators are creation operators, while the operators $C_{s}(z)$ acts on $|\Omega\rangle$ as the annihilation operators. This motivates us to consider the states

$$
\left.\left|v_{1}, v_{2}, \ldots v_{n}\right| F\right\rangle_{0}=F^{a_{n} \ldots a_{1}} B_{0, a_{1}}\left(v_{1}\right) B_{1, a_{2}}\left(v_{2}\right) \cdots B_{0, a_{n}}\left(v_{n}\right)|\Omega\rangle_{0}, \quad a_{i}=1,2
$$

as $n$-particle eigenstates of the transfer matrix $\tau(u) . F^{a_{n} \ldots a_{1}}$ is a function of the spectral parameters $v_{j}$, which should be found later.

In order to proceed further we need to rewrite the YBEs (18) and (19) in terms of the matrix elements of the monodromy matrix

$$
\begin{gathered}
(-1)^{p\left(k^{\prime \prime}\right)\left(p\left(m^{\prime}\right)+p\left(m^{\prime \prime}\right)\right)} \check{R}_{k^{\prime} m^{\prime}}^{k m}(\bar{u}, v) M_{1 m^{\prime \prime}}^{m^{\prime}}(u) M_{0 k^{\prime \prime}}^{k^{\prime}}(v) \\
=(-1)^{p\left(k^{\prime}\right)\left(p(m)+p\left(m^{\prime}\right)\right)} M_{1 m^{\prime}}^{m}(v) M_{0 k^{\prime}}^{k}(u) \check{R}_{k^{\prime \prime} m^{\prime \prime}}^{k^{\prime} m^{\prime}}(\bar{u}, v), \\
(-1)^{p\left(k^{\prime \prime}\right)\left(p\left(m^{\prime}\right)+p\left(m^{\prime \prime}\right)\right)}\left(\check{\tilde{R}}^{\iota_{1}}\right)_{k^{\prime} m^{\prime}}^{k m}(\bar{u}, v) M_{0 m^{\prime \prime}}^{m^{\prime}}(u) M_{1_{k^{\prime \prime}}}^{k^{\prime}}(v) \\
=(-1)^{p\left(k^{\prime}\right)\left(p(m)+p\left(m^{\prime}\right)\right)} M_{0 m^{\prime}}^{m}(v) M_{k^{\prime}}^{k}(u)\left(\tilde{\tilde{R}}^{\iota_{1}}\right)_{k^{\prime \prime} m^{\prime \prime}}^{k^{\prime} m^{\prime}}(\bar{u}, v) .
\end{gathered}
$$

For our purpose, the interesting components of the equations (52) are the following commutation relations between the operators $A_{b c}(u)$ and $D(u)$ with the $B_{c}(v)$

$$
\begin{aligned}
A_{1, b a}(u) B_{0, c}(v) & =\frac{r_{b c}^{b^{\prime} c^{\prime}}(u, v)}{b(u, v)} B_{1, c^{\prime}}(v) A_{0, b^{\prime} a}(u)+\frac{a_{a}(u, v)}{b(u, v)} B_{1, b}(u) A_{0, c a}(v), \\
A_{0, b a}(u) B_{1, c}(v)= & -\frac{r_{b c}^{b^{\prime} c^{\prime}}(u, v)}{b(u, v)} B_{0, c^{\prime}}(v) A_{1, b^{\prime} a}(u)-\frac{a_{a}(u, v)}{b(u, v)} B_{0, b}(u) A_{1, c a}(v), \\
D_{1}(u) B_{0, a}(v) & =\frac{1}{b^{\prime}(u, v)} B_{1, a}(v) D_{0}(u)-\frac{a_{a}^{\prime}(v, u)}{b^{\prime}(v, u)} B_{1, a}(u) D_{0}(v), \\
D_{0}(u) B_{1, a}(v) & =-\frac{1}{b^{\prime}(u, v)} B_{0, a}(v) D_{1}(u)+\frac{a_{a}^{\prime}(v, u)}{b^{\prime}(v, u)} B_{0, a}(u) D_{1}(v), \\
B_{1, a}(u) B_{0, b}(v) & =r_{a b}^{b^{\prime} a^{\prime}}(u, v) B_{1, a^{\prime}} B_{0, b^{\prime}}(u),
\end{aligned}
$$




$$
B_{0, a}(u) B_{1, b}(v)=r_{a b}^{b^{\prime} a^{\prime}}(u, v) B_{0, a^{\prime}} B_{1, b^{\prime}}(u),
$$

where we have defined $b(u, v), a_{a}(u, v), b^{\prime}(u, v), a_{a}^{\prime}(v, u)$ as follows

$$
\begin{aligned}
b(u, v) & =z(u, v)-z^{-1}(u, v), \\
a_{a}(u, v) & =\left\{\begin{array}{l}
-\lambda z(u, v), \quad \text { for } \quad a=1, \\
-\lambda z^{-1}(u, v), \quad \text { for } \quad a=2,
\end{array}\right. \\
b^{\prime}(u, v) & =-\frac{z(u, v)-z^{-1}(u, v)}{q^{-1} z^{-1}(u, v)-q z(u, v)}, \\
a_{a}^{\prime}(v, u) & = \begin{cases}\frac{\lambda z(u, v)}{q^{-1} z(u, v)-q z^{-1}(u, v)}, & \text { for } \quad a=1, \\
\frac{\lambda z^{-1}(u, v)}{q^{-1} z(u, v)-q z^{-1}(u, v)}, & \text { for } \quad a=2\end{cases}
\end{aligned}
$$

and

$$
\begin{aligned}
& r_{b c}^{b^{\prime} c^{\prime}}(u, v)= \\
& \left(\begin{array}{cccc}
q z(u, v)-q^{-1} z^{-1}(u, v) & 0 & 0 & 0 \\
0 & z(u, v)-z^{-1}(u, v) & \lambda z^{-1}(u, v) & 0 \\
0 & \lambda z(u, v) & z(u, v)-z^{-1}(u, v) & 0 \\
0 & 0 & 0 & q z(u, v)-q^{-1} z^{-1}(u, v)
\end{array}\right) .
\end{aligned}
$$

Here we have used the notation $z(u, v)=\exp (i[u-v])=\frac{z(u)}{z(v)}$.

The first terms in the commutation relations (53)-(56) are so called "wanted" terms. As we will see they are contributing to the eigenvalues of the transfer matrix $\tau_{s}(u), s=0,1$. The second terms in the (53)-(56) are so called "unwanted" terms and their contribution should be canceled in order to have an eigenstate.

Using (53)-(58) we can represent the action of the diagonal elements of the monodromy matrix as follows

$$
\begin{aligned}
& \left.D_{1}(u)\left|v_{1}, \ldots, v_{n}\right| F\right\rangle_{1}= \\
& \left.(-1)^{\frac{n}{2}} \prod_{i=1}^{n} \frac{1}{b^{\prime}\left(\bar{u}, v_{i}\right)}\left[q^{-1} z^{-1}(u)-q z(u)\right]^{\frac{N}{2}}\left[h q^{-1} z^{-1}(u)-h^{-1} q z(u)\right]^{\frac{N}{2}}\left|v_{1}, \ldots, v_{n}\right| F\right\rangle_{0} \\
& +\sum_{k=1}^{n}\left(\tilde{\Lambda}_{k}\right)_{a_{1} \ldots a_{n}}^{b_{1} \ldots b_{n}} F^{a_{n} \ldots a_{1}} B_{1, b_{k}}(\bar{u}) \prod_{j=1, j \neq k}^{n} B_{b_{j}}\left(v_{j}\right)|\Omega\rangle_{1},
\end{aligned}
$$

and

$$
\left.\left[A_{1,11}(u)+A_{1,22}(u)\right]\left|v_{1}, \ldots, v_{n}\right| F\right\rangle_{1}=
$$




$$
\begin{aligned}
= & (-1)^{\frac{n}{2}+1} \prod_{i=1}^{n} \frac{1}{b\left(\bar{u}, v_{i}\right)}\left[-z(u)+z^{-1}(u)\right]^{\frac{N}{2}}\left[h^{-1} z(u)-h z^{-1}(u)\right]^{\frac{N}{2}} . \\
& \tau^{(1)}{ }_{a_{1} \ldots a_{n}}^{a_{1}^{\prime} \ldots a_{n}^{\prime}}\left(\bar{u}, v_{1}, \ldots, v_{n}\right) F^{a_{n} \ldots a_{1}} \prod_{i=1}^{n} B_{a_{i}^{\prime}}\left(v_{i}\right)|\Omega\rangle_{1}+ \\
+ & \sum_{k=1}^{n}\left(\Lambda_{k}\right)_{a_{1} \ldots a_{n}}^{b_{1} \ldots b_{n}} F^{a_{n} \ldots a_{1}} B_{1, b_{k}}(\bar{u}) \prod_{i=1, j \neq k}^{n} B_{b_{j}}\left(v_{j}\right)|\Omega\rangle_{1},
\end{aligned}
$$

where

$$
\tau^{(1)}{ }_{a_{1} \ldots a_{n}}^{a_{1}^{\prime} \ldots a_{n}^{\prime}}\left(\bar{u}, v_{1}, \ldots, v_{n}\right)=-r_{c a_{1}}^{b_{1} a_{1}^{\prime}}\left(\bar{u}, v_{1}\right) r_{b_{1} a_{2}}^{b_{2} a_{2}^{\prime}}\left(\bar{u}, v_{2}\right) \cdots r_{b_{n-1} a_{n}}^{c a_{n}^{\prime}}\left(u, v_{n}\right)
$$

In the equations (61) and (62) the first terms appeared due to the "wanted" terms of the commutation relations (53)-(56) while the second terms result from the "unwanted" terms.

The expression (63) for $\tau^{(1)}{ }_{a_{1} \ldots a_{n}}^{a_{1}^{\prime} \ldots a_{n}^{\prime}}$ can be rewritten as $\operatorname{str}\left[l_{n}\left(\bar{u}, v_{n}\right) l_{(n-1)}\left(\bar{u}, v_{n-1}\right) \ldots l_{1}\left(\bar{u}, v_{1}\right)\right]$, with

$$
\begin{aligned}
{\left[l_{k}\left(\bar{u}, v_{k}\right)\right]_{b_{k-1}}^{b_{k}}=} & r_{b_{k-1} a_{k}}^{b_{k} a_{k}^{\prime}}\left(\bar{u}, v_{k}\right) \\
= & \left(\begin{array}{ll}
{\left[q z\left(\bar{u}, v_{k}\right)-q^{-1} z^{-1}\left(\bar{u}, v_{k}\right)\right] e_{1}^{1}} & \lambda z^{-1}\left(\bar{u}, v_{k}\right) e_{1}^{2} \\
+\left(z\left(\bar{u}, v_{k}\right)-z^{-1}\left(\bar{u}, v_{k}\right)\right) e_{2}^{2} & \\
\lambda z\left(\bar{u}, v_{k}\right) e_{2}^{1} & {\left[q z\left(\bar{u}, v_{k}\right)-q^{-1} z^{-1}\left(\bar{u}, v_{k}\right)\right] e_{2}^{2}} \\
& +\left(z\left(\bar{u}, v_{k}\right)-z^{-1}\left(\bar{u}, v_{k}\right)\right) e_{1}^{1}
\end{array}\right) .
\end{aligned}
$$

Here the quantum operators $e_{a}^{b}$ act on the $k$-th space and have $\left(e_{a}^{b}\right)_{\beta}^{\alpha}=\delta_{a}{ }^{\alpha} \delta_{\beta}{ }^{b}$ matrix representation.

As it follows from the equations(61), 62 and (42), in order $\left.\left|v_{1}, \ldots, v_{n}\right| F\right\rangle$ to be an eigenstate of $\tau_{s}(u)$

$$
\left.\left.\tau_{s}(u)\left|v_{1}, \ldots, v_{n}\right| F\right\rangle=\nu_{s}\left(u ; v_{1}, \ldots, v_{n},\right)\left|v_{1}, \ldots, v_{n}\right| F\right\rangle
$$

we should demand

i) the cancellation of the unwanted terms

$$
\left[\left(\tilde{\Lambda}_{k}\right)_{a_{1} \ldots a_{n}}^{b_{1} \ldots b_{n}}-\left(\Lambda_{k}\right)_{a_{1} \ldots a_{n}}^{b_{1} \ldots b_{n}}\right] F^{a_{n} \ldots a_{1}}=0
$$

and

ii) $F$ should be an eigenvector of the small (nested) transfer matrix $\tau^{(1)}(u)$

$$
\tau^{(1)}{ }_{a_{1} \ldots a_{n}}^{a_{1}^{\prime} \ldots a_{n}^{\prime}}\left(u ; v_{1}, \ldots, v_{n}\right) F^{a_{n} \ldots a_{1}}=\nu^{(1)}\left(u ; v_{1}, \ldots, v_{n}\right) F^{a_{n}^{\prime} \ldots a_{1}^{\prime}}
$$


Once this conditions are fulfilled, the expressions for the eigenvalues of $\tau_{s}(u)$ become

$$
\begin{aligned}
& \nu_{1}\left(u ; v_{1}, \ldots, v_{n}\right)= \\
= & (-1)^{\frac{n}{2}} \prod_{i=1}^{n} \frac{1}{b^{\prime}\left(\bar{u}, v_{i}\right)}\left[q^{-1} z^{-1}(u)-q z(u)\right]^{\frac{N}{2}}\left[h q^{-1} z^{-1}(u)-h^{-1} q z(u)\right]^{\frac{N}{2}} \\
- & (-1)^{\frac{n}{2}} \prod_{i=1}^{n} \frac{1}{b\left(\bar{u}, v_{i}\right)}\left[-z(u)+z^{-1}(u)\right]^{\frac{N}{2}}\left[h^{-1} z(u)-h z^{-1}(u)\right]^{\frac{N}{2}} \nu^{(1)}\left(u ; v_{1}, \ldots, v_{n}\right),
\end{aligned}
$$

with

$$
\nu_{0}(u)=\nu_{1}(\bar{u})
$$

Therefore, as it is obvious from the preceding analysis, in order to know exactly the eigenvalue we need to solve an other eigenvalue-eigenstate problem (67) for the transfer matrix $\tau^{(1)}$ with the reduced amount of degrees of freedom. That is why the all procedure is called Nested Bethe Ansatz $(N B A)$.

In the article [32] the computation of the quantities $\tilde{\Lambda}_{k}$ and $\Lambda_{k}$ for the ordinary $t-J$ model was demonstrated and the condition of cancellation of the unwanted terms was reduced to some equations, which are defining the first set of Bethe equations. It is not necessary to repeat this calculations here, but let us mention the main difference, namely

$$
\begin{gathered}
\left(A_{1,11}+A_{1,22}\right)|\Omega\rangle=\left[-z(u)+z^{-1}(u)\right]^{\frac{N}{2}}\left[h^{-1} z(u)-h z^{-1}(u)\right]^{\frac{N}{2}}|\Omega\rangle \\
D_{1}|\Omega\rangle=\left[-q z(u)+q^{-1} z^{-1}(u)\right]^{\frac{N}{2}}\left[-h^{-1} z(u) q+h z^{-1}(u) q^{-1}\right]^{\frac{N}{2}}|\Omega\rangle
\end{gathered}
$$

which leads to the condition

$$
\begin{aligned}
& \tau^{(1)}{ }_{b_{1} \ldots b_{n}}^{b_{1}^{\prime} \ldots b_{n}^{\prime}}\left(v_{k} ; v_{1}, \ldots, v_{n}\right) F^{b_{n} \ldots b_{1}}=-\lambda \prod_{i=1 ; i \neq k}^{n}\left[q z\left(v_{k}, v_{i}\right)-q^{-1} z^{-1}\left(v_{k}, v_{i}\right)\right] . \\
& \frac{\left[q^{-1} z^{-1}\left(\bar{v}_{k}\right)-q z\left(\bar{v}_{k}\right)\right]^{\frac{N}{2}}\left[h q^{-1} z^{-1}\left(\bar{v}_{k}\right)-h^{-1} q z\left(\bar{v}_{k}\right)\right]^{\frac{N}{2}}}{\left[-z\left(\bar{v}_{k}\right)+z^{-1}\left(\bar{v}_{k}\right)\right]^{\frac{N}{2}}\left[h^{-1} z\left(\bar{v}_{k}\right)-h z^{-1}\left(\bar{v}_{k}\right)\right]^{\frac{N}{2}}} F^{b_{n}^{\prime} \ldots b_{1}^{\prime}} .
\end{aligned}
$$

The next step of NABA is the diagonalization of the small transfer matrix $\tau^{(1)}(u)$ for a chain of length $n$ in order for $F$ to become an eigenvector of it. Following the ABA technique we write the $Y B E$

$$
\check{r}_{k^{\prime} m^{\prime}}^{k m}(u, v) M_{m^{\prime \prime}}^{(1)} \stackrel{m^{\prime}}{(u) M_{k^{\prime \prime}}^{(1)}} \underset{k^{\prime}}{(v)}=M_{m^{\prime}}^{(1)}(v) M_{k^{\prime}}^{m}(u) \check{r}_{k^{\prime \prime} m^{\prime \prime}}^{k^{\prime} m^{\prime}}(u, v)
$$

where $M^{(1)}{ }_{a}^{a^{\prime}}$ is a monodromy matrix for the "nested" problem, and

$$
\check{r}_{b c}^{b^{\prime} c^{\prime}}(u, v)
$$




$$
=\left(\begin{array}{cccc}
q z(u, v)-q^{-1} z^{-1}(u, v) & 0 & 0 & 0 \\
0 & \lambda z(u, v) & z(u, v)-z^{-1}(u, v) & 0 \\
0 & z(u, v)-z^{-1}(u, v) & \lambda z^{-1}(u, v) & 0 \\
0 & 0 & 0 & q z(u, v)-q^{-1} z^{-1}(u, v)
\end{array}\right) .
$$

is the $r$-matrix from $(60)$ in a braid formalism.

Let us now take

$$
M^{(1)}(u)=\left(\begin{array}{ll}
A^{(1)}(u) & B^{(1)}(u) \\
C^{(1)}(u) & D^{(1)}(u)
\end{array}\right)
$$

and the corresponding trace

$$
\tau^{(1)}(u)=-A^{(1)}(u)-D^{(1)}(u) .
$$

From the formulas (72) one can choose following algebraic relations

$$
\begin{aligned}
A^{(1)}(u) B^{(1)}(v)= & \frac{q z(u, v)-q^{-1} z^{-1}(u, v)}{z(u, v)-z^{-1}(u, v)} B^{(1)}(v) A^{(1)}(u) \\
& -\frac{\lambda z(u, v)}{z(u, v)-z^{-1}(u, v)} B^{(1)}(u) A^{(1)}(v), \\
D^{(1)}(u) B^{(1)}(v)= & \frac{q^{-1} z(u, v)-q z^{-1}(u, v)}{z(u, v)-z^{-1}(u, v)} B^{(1)}(v) D^{(1)}(u) \\
& +\frac{\lambda z(u, v)}{z(u, v)-z^{-1}(u, v)} B^{(1)}(u) D^{(1)}(v), \\
B^{(1)}(u) B^{(1)}(v)= & B^{(1)}(v) B^{(1)}(u),
\end{aligned}
$$

which are the $Y B E$ for the $X X Z$ model with staggered inhomogeneity, defined in the article [13. The first (second) term in the expression (76) called "wanted" (correspondingly "unwanted") term.

Let us take

$$
\begin{array}{r}
|0\rangle_{k}^{(1)}=\left(\begin{array}{l}
1 \\
0
\end{array}\right), \\
|\Omega\rangle^{(1)}=|0\rangle_{1}^{(1)} \ldots|0\rangle_{n}^{(1)}=\bigotimes_{k=1}^{n}|0\rangle_{k}^{(1)} .
\end{array}
$$

as a reference state. In order to find the action of nested monodromy matrix $M^{(1)}(u)$ on the reference state one should act by $l_{k}(u)$ from $(64)$ on $|0\rangle_{k}^{(1)}$. 
After simple calculations we obtain

$$
\begin{gathered}
A^{(1)}(u)|\Omega\rangle^{(1)}=\prod_{i=1}^{n}\left[q z\left(u, v_{i}\right)-q^{-1} z^{-1}\left(u, v_{i}\right)\right]|\Omega\rangle^{(1)} \\
D^{(1)}(u)|\Omega\rangle^{(1)}=\prod_{i=1}^{n}\left[z\left(u, v_{i}\right)-z^{-1}\left(u, v_{i}\right)\right]|\Omega\rangle^{(1)} .
\end{gathered}
$$

Now let us make the following Ansatz for the eigenstates of $\tau^{(1)}(u)$

$$
\left|v_{1}^{(1)}, \ldots, v_{m}^{(1)}\right\rangle=B^{(1)}\left(v_{1}^{(1)}\right) B^{(1)}\left(v_{2}^{(1)}\right) \ldots B^{(1)}\left(v_{m}^{(1)}\right)|\Omega\rangle^{(1)} .
$$

By use of $(\sqrt{76})$ the actions of $A^{(1)}(u)$ and $D^{(1)}(u)$ on the states $(\sqrt{79})$ is given by

$$
\begin{aligned}
D^{(1)}(u) \mid & \left.v_{1}^{(1)}, \ldots, v_{m}^{(1)}\right\rangle \\
= & \prod_{j=1}^{m} \frac{q^{-1} z\left(u, v_{i}^{(1)}\right)-q z^{-1}\left(u, v_{i}^{(1)}\right)}{z\left(u, v_{i}^{(1)}\right)-z^{-1}\left(u, v_{i}^{(1)}\right)} \prod_{j=1}^{n}\left[z\left(u, v_{j}\right)-z^{-1}\left(u, v_{j}\right)\right]\left|v_{1}^{(1)}, \ldots, v_{m}^{(1)}\right\rangle \\
& +\sum_{k=1}^{m} \Lambda_{k}^{(1)} B^{(1)}(u) \prod_{j=1, j \neq k}^{m} B^{(1)}\left(v_{j}^{(1)}\right)|\Omega\rangle^{(1)}, \\
A^{(1)}(u) \mid & \left.v_{1}^{(1)}, \ldots, v_{m}^{(1)}\right\rangle \\
= & \prod_{i=1}^{m} \frac{q z\left(u, v_{i}^{(1)}\right)-q^{-1} z^{-1}\left(u, v_{i}^{(1)}\right)}{z\left(u, v_{i}^{(1)}\right)-z^{-1}\left(u, v_{i}^{(1)}\right)} \prod_{j=1}^{n}\left[q z\left(u, v_{i}\right)-q^{-1} z^{-1}\left(u, v_{i}\right)\right]\left|v_{1}^{(1)}, \ldots, v_{m}^{(1)}\right\rangle \\
& +\sum_{k=1}^{m} \tilde{\Lambda}_{k}^{(1)} B^{(1)}(u) \prod_{j=1, j \neq k}^{m} B^{(1)}\left(v_{j}^{(1)}\right)|\Omega\rangle^{(1)} .
\end{aligned}
$$

The first terms in this expressions are the "wanted" terms, while the second terms (with $\Lambda_{k}^{(1)}, \tilde{\Lambda}_{k}^{(1)}$ ) are the "unwanted" terms of the nested problem and should cancel each other in the eigenvalue equation (67). Hence we can write the eigenvalues $\nu^{(1)\left(u ; v_{1}, \ldots, v_{n} ; v_{1}^{(1)}, \ldots, v_{m}^{(1)}\right)}$ of $\tau^{(1)}(u)$

$$
\begin{array}{r}
\tau^{(1)}(u)\left|v_{1}^{(1)}, \ldots, v_{m}^{(1)}\right\rangle=\left[-A^{(1)}(u)-D^{(1)}(u)\right]\left|v_{1}^{(1)}, \ldots, v_{m}^{(1)}\right\rangle \\
=-\left[\prod_{i=1}^{m} \frac{q z\left(u, v_{i}^{(1)}\right)-q^{-1} z^{-1}\left(u, v_{i}^{(1)}\right)}{z\left(u, v_{i}^{(1)}\right)-z^{-1}\left(u, v_{i}^{(1)}\right)} \prod_{j=1}^{n}\left[q z\left(u, v_{i}\right)-q^{-1} z^{-1}\left(u, v_{i}\right)\right]\right. \\
\left.+\prod_{j=1}^{m} \frac{q^{-1} z\left(u, v_{i}^{(1)}\right)-q z^{-1}\left(u, v_{i}^{(1)}\right)}{z\left(u, v_{i}^{(1)}\right)-z^{-1}\left(u, v_{i}^{(1)}\right)} \prod_{j=1}^{n}\left[z\left(u, v_{j}\right)-z^{-1}\left(u, v_{j}\right)\right]\right]\left|v_{1}^{(1)}, \ldots, v_{m}^{(1)}\right\rangle .
\end{array}
$$


Setting now $u=v_{k}$ in (81) and comparing it with the condition (71) we obtain the first set of Bethe equations

$$
\begin{aligned}
\prod_{i=1}^{m} \frac{q z\left(v_{k}, v_{i}^{(1)}\right)-q^{-1} z^{-1}\left(v_{k}, v_{i}^{(1)}\right)}{z\left(v_{k}, v_{i}^{(1)}\right)-z^{-1}\left(v_{k}, v_{i}^{(1)}\right)} & =\frac{\left[h^{-1} q^{-1} z\left(v_{k}\right)-h q z^{-1}\left(v_{k}\right)\right]^{\frac{N}{2}}\left[q^{-1} z\left(v_{k}\right)-q z^{-1}\left(v_{k}\right)\right]^{\frac{N}{2}}}{\left[-h^{-1} z\left(v_{k}\right)+h z^{-1}\left(v_{k}\right)\right]^{\frac{N}{2}}\left[z\left(v_{k}\right)-z^{-1}\left(v_{k}\right)\right]^{\frac{N}{2}}} \\
k & =1, \ldots, n .
\end{aligned}
$$

The equations which are obtained from the cancellation of the unwanted terms $\Lambda_{k}^{(1)}$ and $\tilde{\Lambda}_{k}^{(1)}$

$$
\begin{aligned}
& \prod_{i=1, i \neq k}^{m} \frac{q^{-1} z\left(v_{k}^{(1)}, v_{i}^{(1)}\right)-q z^{-1}\left(v_{k}^{(1)}, v_{i}^{(1)}\right)}{q z\left(v_{k}^{(1)}, v_{i}^{(1)}\right)-q^{-1} z^{-1}\left(v_{k}^{(1)}, v_{i}^{(1)}\right)}=\prod_{j=1}^{n} \frac{q z\left(v_{k}^{(1)}, v_{j}\right)-q^{-1} z^{-1}\left(v_{k}^{(1)}, v_{j}\right)}{z\left(v_{k}^{(1)}, v_{j}\right)-z^{-1}\left(v_{k}^{(1)}, v_{j}\right)} \\
& k=1 \ldots, m
\end{aligned}
$$

are the second set of Bethe equations.

Finally we obtain the eigenvalues of $\tau_{1}(u)$ and $\tau_{0}(u)$ from $(68)$ and (69) as

$$
\begin{aligned}
\nu_{1}\left(u ;\{v\} ;\left\{v^{(1)}\right\}\right)= & (-1)^{\frac{n}{2}} \prod_{i=1}^{n} \frac{1}{b^{\prime}\left(\bar{u}, v_{i}\right)}\left[q^{-1} z^{-1}(u)-q z(u)\right]^{\frac{N}{2}}\left[h q^{-1} z^{-1}(u)-h^{-1} q z(u)\right]^{\frac{N}{2}} \\
& +(-1)^{\frac{n}{2}} \prod_{i=1}^{n} \frac{1}{b\left(\bar{u}, v_{i}\right)}\left[-z(u)+z^{-1}(u)\right]^{\frac{N}{2}}\left[h^{-1} z(u)-h z^{-1}(u)\right]^{\frac{N}{2}} . \\
& \cdot\left[\prod_{i=1}^{m} \frac{q z\left(u, v_{i}^{(1)}\right)-q^{-1} z^{-1}\left(u, v_{i}^{(1)}\right)}{z\left(u, v_{i}^{(1)}\right)-z^{-1}\left(u, v_{i}^{(1)}\right)} \prod_{j=1}^{n}\left[q z\left(u, v_{i}\right)-q^{-1} z^{-1}\left(u, v_{i}\right)\right]\right. \\
& \left.+\prod_{j=1}^{m} \frac{q^{-1} z\left(u, v_{i}^{(1)}\right)-q z^{-1}\left(u, v_{i}^{(1)}\right)}{z\left(u, v_{i}^{(1)}\right)-z^{-1}\left(u, v_{i}^{(1)}\right)} \prod_{j=1}^{n}\left[z\left(u, v_{j}\right)-z^{-1}\left(u, v_{j}\right)\right]\right]
\end{aligned}
$$

and

$$
\nu_{0}\left(u ;\{v\} ;\left\{v^{(1)}\right\}\right)=\nu_{1}\left(\bar{u} ;\{v\} ;\left\{v^{(1)}\right\}\right) .
$$

\section{Acknowledgements}

The author acknowledge D.Arnaudon, A.Sedrakyan, P.Sorba for the formulation of the problem and many fruitful discussions.

Work was partially supported by INTAS grant 99-1459. 


\section{References}

[1] E. Dagotto, T.M. Rice, Science 271(1996) 618,

[2] J. Baxter, Exactly Solved Models in Statistical Mechanics, Academic Press, London (1989)

[3] L. Faddeev, L. Takhtajian - Russian Math.Surveys 34:5(1979)11

V. Korepin, N.M. Bogoliubov, A. Izergin - Quantum Inverse Scattering Method and Correlation Functions - Cambridge Univ.Press - 1993.

[4] Y. Wang, Phys.Rev. B 60(1999) 9236,

M. Batchelor, M. Maslen, J. Phys. A 32(1999) L377,

M. Batchelor, J. de Gier, J. Links and M. Maslen, J. Phys. A 33(2000) L97,

[5] A. Kolezhuk, H.-J. Mikeska, Int.J. Mod.Phys. B 12(1998) 2325,

A. Honecker,F. Mila,M. Troyer, cond-mat/9910438,

[6] N. Muramoto, M. Takahashi, J. Phys.Soc.Japan 68(1999) 2098,

[7] C.K. Majumdar, D.K. Ghosh - J. Math.Phys.10(1969) 1399,

[8] H.J. DeVega, F. Woynarovich - J. Phys.A 25(1992) 4499,

H.J. DeVega, L. Mezincescu, R. Nepomechie - Phys.Rev.49 B(1994) 13223,

[9] St. Meißner, B-D, Dörfel - J. Phys.A 29(1996) 1949, 6471

[10] S. Albeverio, S.-M. Fei, Y. Wang, Europhys.Lett. 47(1999) 364,

[11] J. Links, A. Foerster, Solution of a two leg spin ladder system, cond-mat/9911096,

[12] C. Destri, H.J. DeVega - Nucl.Phys. 290B(1987) 363,

[13] D. Arnaudon, R. Poghossian, A. Sedrakyan, P. Sorba, Nucl. Phys. 588 B [FS](2000) 638 ,

D. Arnaudon, R. Poghossian, A. Sedrakyan, T. Sedrakyan, and P. Sorba, "Construction of integrable models on ladders, and related quantum symmetries," in Non-perturbative Quantum Effects 2000, Paris, September 7-13, 2000 (B. Julia and D. Bernard, eds.), JHEP vol. PRHEP-tmr2000/053.

[14] J. Ambjorn, D. Arnaudon, A. Sedrakyan, T. Sedrakyan, P. Sorba, J. Phys. A: Math. Gen. 34 (2001) 5887-5900,

[15] D. Arnaudon, A. Sedrakyan, T. Sedrakyan, P. Sorba, - hep-th/0106139

[16] A. Kavalov, A. Sedrakyan, Nucl. Phys. 285 B [FS19] (1987) 264, 
[17] A. Sedrakyan, Nucl.Phys. 554 B [FS] (1999) 514,

[18] L.D. Faddeev - How Algebraic Bethe Ansatz works for integrable models, hep-th/9605187,

[19] V. Yu. Popkov, A.A. Zvyagin, Phys.Lett. A 175 (1993) 295,

A.A. Zvyagin, Phys.Rev. 51 B (1995) 12579,

A.A. Zvyagin, Low Temp. Phys.26 (2000) 134,

[20] A.A. Zvyagin, Phys.Rev. 52 B (1995) 15050,

A.A. Zvyagin, Phys.Rev. 57 B (1998) 1035,

[21] H. Frahm, C. Rödenbeck, Europhys.Lett. 33 (1996) 47,

[22] X.G.Wen, F. Wilczek, A. Zee- Phys.Rev. 39 B(1989) 11413,

[23] J.H.H. Perk, C.L. Schultz, Phys.Lett. 84 A(1981) 407,

C.L. Schultz Phys.Rev.Lett. 46(1981) 629,

[24] H.J.de Vega, E. Lopes, Phys.Rev.Lett. 67(1991) 489,

[25] D. Arnaudon, C. Chryssomalakos, L. Frappat, J. Math. Phys. 36(10) (1995) 5262 ,

[26] T. Hakobyan, A. Sedrakyan - Phys.Lett.377B(1996)250,

A. Avakyan, T. Hakobyan, A. Sedrakyan - Nucl.Phys.490B [FS] (1997)633,

J. Ambjorn, A. Avakyan, T. Hakobyan, A. Sedrakyan - Mod.Phys.Let. A13 (1998) 495 ,

J. Ambjorn, A. Avakyan, T. Hakobyan, A. Sedrakyan - J. Math.Phys. 41 (1999)

[27] J. Ambjorn, D. Karakhanyan, M. Mirumyam, A. Sedrakyan - Nucl.Phys. 599B [FS](2001) 547,

[28] G. Uimin - JETP Lett. 12 (1970)225

[29] J.K. Lai - J. Math.Phys. 15(1974) 1675

[30] B. Sutherland - Phys.Rev. B12 (1975) 3795

[31] A. Foerster, M. Karowski- Nucl.Phys.408 B [FS](1993)512,

A. Foerster, PhD Thesis(FU-Berlin, 1993),

H. J.deVega, A. Gonzales-Ruiz-Nucl.Phys.417 B [FS](1994)553,

A. Gonzales-Ruiz-Nucl.Phys.424 B [FS](1994)468,

Rui-Hong Yue, Heng Fan, Bo-yu Hou-Nucl. Phys.462 B(1996)167,

[32] F.H.L. Essler, V. Korepin - Phys.Rev.46B(1992)9247 